\title{
Optimal feedback control of two-qubit entanglement in dissipative environments
}

\author{
Morteza Rafiee, ${ }^{1, *}$ Alireza Nourmandipour, ${ }^{2, \dagger}$ and Stefano Mancini ${ }^{3,4, \ddagger}$ \\ ${ }^{1}$ Department of Physics, Shahrood University of Technology, 3619995161 Shahrood, Iran \\ ${ }^{2}$ Atomic and Molecular Group, Faculty of Physics, Yazd University, 89195-741 Yazd, Iran \\ ${ }^{3}$ School of Science \& Technology, University of Camerino, I-62032 Camerino, Italy \\ ${ }^{4}$ INFN, Sezione di Perugia, Via A. Pascoli, I-06123 Perugia, Italy
}

(Received 7 April 2016; published 6 July 2016)

\begin{abstract}
We study the correction of errors intervening in two qubits dissipating into their own environments. This is done by resorting to local feedback actions with the aim of preserving as much as possible the initial amount of entanglement. Optimal control is found first by gaining insights from the subsystem purity and then by numerical analysis on the concurrence. This is tantamount to a double optimization on the actuation and on the measurement processes. Repeated feedback action is also investigated, thus paving the way for a continuous-time formulation and a solution of the problem.
\end{abstract}

DOI: 10.1103/PhysRevA.94.012310

\section{INTRODUCTION}

The feature of quantum mechanics that distinguishes it most from classical mechanics is the coherent superposition of distinct physical states, usually referred to as quantum coherence. It embraces also entanglement, i.e., nonlocal quantum correlations arising in composite systems [1]. Quantum coherence results are rather fragile against environment effects and this fact has boosted the development of a quantum control theory [2]. Just like the classical one, quantum control theory includes open-loop control and closed-loop control according to the principle of controller design [3]. Feedback is a paradigm of closed-loop control in that it involves gathering information about the system state and then, according to that, actuates a corrective action on its dynamics. It has been shown that quantum feedback is superior to open-loop control in dealing with uncertainties in initial states [4]. Moreover, it has been proven that it works better than open-loop control when it aims at restoring quantum coherence [5].

In the presence of feedback, suitable quantum operations are added to the bare dynamical map (resulting from the environment action) of a quantum system. These quantum operations should be determined according to the desired target state, that is, one optimizes the actuation. In addition, it is known that there is a correspondence between measurement on the environment and the representation of the map [6]. Therefore, it is clear that one has to optimize the measurement over all possible representations of the map in order to extract the maximum information with the minimum disturbance. Altogether, it can be said that feedback implies in the quantum realm a double optimization over the measurement and over the actuation process [7]. This makes designing the optimal feedback control a daunting task for quantum systems, especially composite ones and hence entanglement control (we refer here to local control, i.e., measurement and actuation are both local operations). In linear bosonic systems the pursued strategy was to steer a system towards a stationary state

\footnotetext{
*m.rafiee178@gmail.com

${ }^{\dagger}$ Corresponding author: anoormandip@ stu.yazd.ac.ir

${ }^{\ddagger}$ stefano.mancini@unicam.it
}

entangled as much as possible [8]. Dealing with the inherent nonlinearity of qubits makes this strategy very challenging and no progress has been made since the seminal work of Ref. [9].

Hence, we consider here a feedback control whose aim is to preserve as much as possible an initial maximally entangled state for two qubits dissipating into their own environments. Actually, we employ maps and corrective actions much in the spirit of [10], without analyzing continuous-time evolution. Optimal control is found by first gaining insights from the subsystem purity and then by numerical analysis of the concurrence. Repeated feedback action is also investigated, thus paving the way for a continuous-time formulation and a solution of the problem.

The layout of the paper is as follows. We start by introducing the model in Sec. II. Then we discuss the feedback action in Sec. III and subsequently address its optimality in Sec. IV. Section $\mathrm{V}$ is devoted to repeated applications of the dynamical map. We summarize in Sec. VI.

\section{MODEL}

We consider two qubits (distinguished whenever necessary by labels $A$ and $B$ ) undergoing the effect of local amplitude damping so that their initial state $\rho$ changes according to the quantum channel map

$$
\rho \mapsto \rho^{\prime}=\sum_{j=1}^{4} K_{j} \rho K_{j}^{\dagger},
$$

where

$$
\begin{aligned}
& K_{1}=E_{1} \otimes E_{1}, \quad K_{2}=E_{1} \otimes E_{2}, \\
& K_{3}=E_{2} \otimes E_{1}, \quad K_{4}=E_{2} \otimes E_{2}
\end{aligned}
$$

are the Kraus operators (satisfying $\sum_{i=1}^{4} K_{i}^{\dagger} K_{i}=I$ ) constructed from those of local (single-qubit) amplitude damping channels

$$
\begin{aligned}
& E_{1}=(\sqrt{\eta}|1\rangle\langle 1|+| 0\rangle\langle 0|), \\
& E_{2}=(\sqrt{1-\eta}|0\rangle\langle 1|) .
\end{aligned}
$$


Here $|0\rangle(|1\rangle)$ is the ground (excited) qubit state and $\eta \in[0,1]$ is the single-qubit damping rate.

The map (1) implies, for each qubit, the probability of losing independently the excitation into its own environment. Suppose that the two qubits are initially prepared in a maximally entangled state, e.g., $\rho=|\Phi\rangle\langle\Phi|$, with

$$
|\Phi\rangle:=\frac{|00\rangle+|11\rangle}{\sqrt{2}} .
$$

In the computational basis $\mathfrak{B}:=\{|11\rangle,|10\rangle,|01\rangle,|00\rangle\}$, it has the following matrix representation:

$$
\rho=\frac{1}{2}\left(\begin{array}{llll}
1 & 0 & 0 & 1 \\
0 & 0 & 0 & 0 \\
0 & 0 & 0 & 0 \\
1 & 0 & 0 & 1
\end{array}\right)
$$

From here on we assume the freedom to perform local operations (and eventually classical communication), i.e., ones that are costless. Hence the above assumption of the initial state is equivalent to any other maximally entangled state.

In the computational basis $\mathfrak{B}$, the state $\rho^{\prime}$ resulting from Eq. (1) reads

$$
\rho^{\prime}=\frac{1}{2}\left(\begin{array}{cccc}
\eta^{2} & 0 & 0 & \eta \\
0 & \eta(1-\eta) & 0 & 0 \\
0 & 0 & \eta(1-\eta) & 0 \\
\eta & 0 & 0 & 2+\eta(\eta-2)
\end{array}\right)
$$

Now consider the subsystem purity

$$
\mathcal{P}(\rho):=\operatorname{Tr}\left(\rho_{A}^{2}\right), \quad \rho_{A}:=\operatorname{Tr}_{B} \rho,
$$

as a measure of entanglement. Although it is only valid for pure states $\rho$, it can give us some insights also into mixed-state entanglement. Due to (6) it is straightforward to show that

$$
\mathcal{P}\left(\rho^{\prime}\right)=\frac{1}{2}\left(2-2 \eta+\eta^{2}\right) .
$$

A minimum of $1 / 2$ is achieved for $\eta=1$, i.e., when the channel (1) reduces to the identity map.

A faithful measure of entanglement is the concurrence defined as [11]

$$
C(\rho):=\max \left\{0, \lambda_{1}-\lambda_{2}-\lambda_{3}-\lambda_{4}\right\}
$$

where $\lambda_{i} \mathrm{~s}$ are, in decreasing order, the non-negative square roots of the moduli of the eigenvalues of $\rho\left(\sigma_{A}^{y} \otimes \sigma_{B}^{y}\right) \rho^{*}\left(\sigma_{A}^{y} \otimes\right.$ $\left.\sigma_{B}^{y}\right)$, with $\rho^{*}$ denoting the complex conjugate of $\rho$. Using (6) we can show that

$$
C\left(\rho^{\prime}\right)=\eta^{2} \sqrt{2+\eta^{2}-2 \eta}-\frac{1}{2} \eta^{2}\left(1-\eta^{2}\right) .
$$

Figure 1 illustrates the subsystem purity as well as the concurrence resulting from the state (1) as a function of $\eta$. We can see that they have opposite behavior. Hence we can argue that parameters minimizing the subsystem purity would also maximize the concurrence.

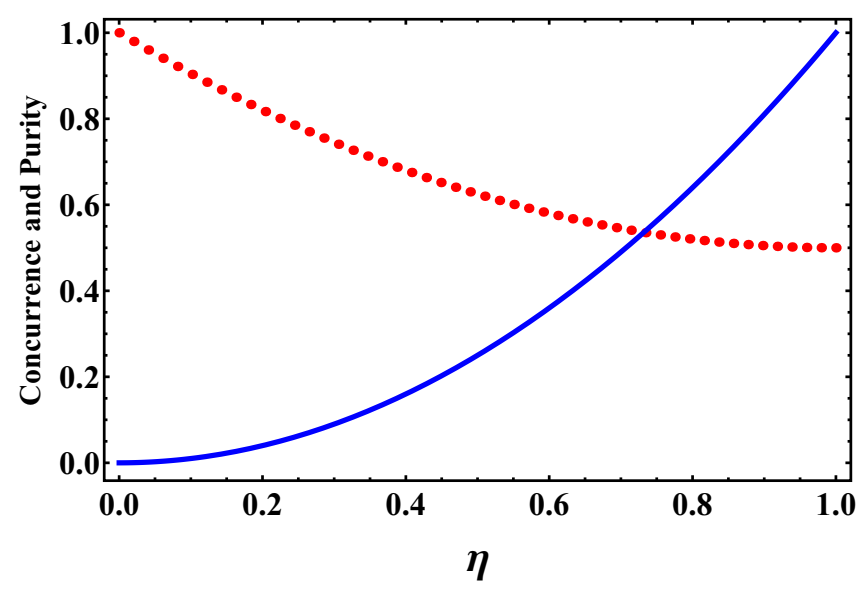

FIG. 1. Concurrence (solid blue line) and subsystem purity (dotted red line) versus $\eta$ for the state $\rho^{\prime}$.

\section{FEEDBACK ACTION}

The map in Eq. (1) can be regarded as the effect of a measurement process described by a probability operator valued measure whose elements are $\left\{K_{j}\right\}_{j}$ and whose outcomes are labeled by the values of $j$ [6]. Notice that the elements $\left\{K_{j}\right\}_{j}$ are local, hence we consider local feedback actions $U_{j} \in \mathrm{SU}(2) \times \mathrm{SU}(2)$ to be applied in correspondence with the outcomes $j$. That is, in the presence of feedback the dynamical map (1) changes into

$$
\rho \mapsto \rho^{\prime \prime}=\sum_{j=1}^{4}\left(U_{j} K_{j}\right) \rho\left(U_{j} K_{j}\right)^{\dagger}
$$

Due to the symmetry of the initial state $\rho=|\Phi\rangle\langle\Phi|$ and of the action of the dissipative map, the unitary operators $U_{j}$ can be taken as

$$
\begin{aligned}
& U_{1}=u \otimes u, \\
& U_{3}=v \otimes u,
\end{aligned}
$$

where

$$
\begin{aligned}
& u=\left(\begin{array}{cc}
e^{-i\left(\phi_{u}+\psi_{u}\right) / 2} \cos \left(\theta_{u} / 2\right) & -e^{-i\left(\phi_{u}-\psi_{u}\right) / 2} \sin \left(\theta_{u} / 2\right) \\
e^{i\left(\phi_{u}-\psi_{u}\right) / 2} \sin \left(\theta_{u} / 2\right) & e^{i\left(\phi_{u}+\psi_{u}\right) / 2} \cos \left(\theta_{u} / 2\right)
\end{array}\right), \\
& v=\left(\begin{array}{cc}
e^{-i\left(\phi_{v}+\psi_{v}\right) / 2} \cos \left(\theta_{v} / 2\right) & -e^{-i\left(\phi_{v}-\psi_{v}\right) / 2} \sin \left(\theta_{v} / 2\right) \\
e^{i\left(\phi_{v}-\psi_{v}\right) / 2} \sin \left(\theta_{v} / 2\right) & e^{i\left(\phi_{v}+\psi_{v}\right) / 2} \cos \left(\theta_{v} / 2\right)
\end{array}\right)
\end{aligned}
$$

are generic elements of $\mathrm{SU}(2)$ with $\phi_{\bullet}, \psi_{\bullet}$, and $\theta_{\bullet}$ the Euler angles.

This model makes complete sense because once we are given an entangled state the feedback operations are completely local and the aim is to restore as much as possible entanglement (degraded by the local dissipation). So the goal is to find the Euler angles that maximize the amount of entanglement of $\rho^{\prime \prime}$. 
Applying (11), with (12) and (13), to $\rho=|\Phi\rangle\langle\Phi|$ gives $\rho^{\prime \prime}$, whose matrix elements in the basis $\mathfrak{B}$ are

$$
\begin{aligned}
{\left[\rho^{\prime \prime}\right]_{11}=} & \frac{1}{8}\left\{\left(1-\eta^{2}\right)\left(1+\cos \theta_{v}\right)^{2}+\left(1+\cos \theta_{u}\right)^{2}\right. \\
& +8 \eta(1-\eta) \cos ^{2}\left(\theta_{v} / 2\right) \sin ^{2}\left(\theta_{u} / 2\right)+4 \eta^{2} \sin ^{4}\left(\theta_{u} / 2\right) \\
& \left.+4 \eta\left(1+\cos \theta_{u}\right) \cos \left(2 \psi_{u}\right) \sin ^{2}\left(\theta_{u} / 2\right)\right\} \\
{\left[\rho^{\prime \prime}\right]_{12}=} & \frac{1}{8}\left(e^{-i \phi_{v}}(1-\eta) \sin \theta_{v}\left[(1-\eta) \cos \theta_{v}+1-\eta \cos \theta_{u}\right]\right. \\
& +e^{-i \phi_{u}} \sin \theta_{u}\left\{(1-\eta)\left(1-\eta \cos \theta_{v}\right)+2 i \eta \sin \left(2 \psi_{u}\right)\right. \\
& \left.\left.+\cos \theta_{u}\left[1+\eta^{2}-2 \eta \cos \left(2 \psi_{u}\right)\right]\right\}\right) \\
& \quad\left[\rho^{\prime \prime}\right]_{13}=\left[\rho^{\prime \prime}\right]_{12}, \\
{\left[\rho^{\prime \prime}\right]_{14}=} & \frac{1}{8} e^{-2 i\left(\phi_{u}+\psi_{u}\right)}\left\{\eta\left(1+\cos \theta_{u}\right)^{2}+4 \eta e^{4 i \psi_{u}} \sin ^{2}\left(\theta_{u} / 2\right)\right. \\
& +2 e^{2 i \psi_{u}}\left(1+\eta^{2}\right)\left(1+\cos \theta_{u}\right) \sin ^{2}\left(\theta_{u} / 2\right) \\
& +2 e^{2 i\left(\phi_{u}-\phi_{v}+\psi_{u}\right)}(1-\eta)^{2}\left(1+\cos \theta_{v}\right) \sin ^{2}\left(\theta_{v} / 2\right) \\
& \left.-2 e^{i\left(\phi_{u}-\phi_{v}+2 \psi_{u}\right)} \eta(1-\eta) \sin _{u} \sin _{v}\right\} \\
{\left[\rho^{\prime \prime}\right]_{22}=} & \frac{1}{8}\left\{4(1-\eta) \eta \cos ^{2}\left(\theta_{u} / 2\right) \cos { }^{2}\left(\theta_{v} / 2\right)\right. \\
& +\left(1+\eta^{2}-2 \eta \cos \left(2 \psi_{u}\right)\right) \sin ^{2} \theta_{u} \\
& \left.+2(1-\eta)\left[1-\eta \cos \theta_{u}+(1-\eta) \cos \theta_{v}\right] \sin ^{2}\left(\theta_{v} / 2\right)\right\} \\
& +17) \\
& +15) \\
&
\end{aligned}
$$

$$
\begin{gathered}
{\left[\rho^{\prime \prime}\right]_{23}=\frac{1}{8}\left\{\left[1+\eta^{2}-2 \eta \cos \left(2 \psi_{u}\right)\right] \sin ^{2} \theta_{u}\right.} \\
\quad-(1-\eta) \sin \theta_{v}\left[2 \eta \cos \left(\phi_{u}-\phi_{v}\right) \sin \theta_{u}\right. \\
\left.\left.\quad-(1-\eta) \sin \theta_{v}\right]\right\} \\
{\left[\rho^{\prime \prime}\right]_{24}=\frac{1}{8}\left(e ^ { - i \phi _ { u } } \operatorname { s i n } \theta _ { u } \left\{(1-\eta)\left(1+\eta \cos \theta_{v}\right)\right.\right.} \\
\left.-\cos \theta_{u}\left[1+\eta^{2}-2 \eta \cos \left(2 \psi_{u}\right)\right]-2 i \eta \sin \left(2 \psi_{u}\right)\right\} \\
\left.+e^{-i \phi_{v}}(1-\eta)\left[1+\eta \cos \theta_{u}-(1-\eta) \cos \theta_{v}\right] \sin \theta_{v}\right) \\
\quad\left[\rho^{\prime \prime}\right]_{33}=\left[\rho^{\prime \prime}\right]_{22}, \\
\quad\left[\rho^{\prime \prime}\right]_{34}=\left[\rho^{\prime \prime}\right]_{24}, \\
{\left[\rho^{\prime \prime}\right]_{44}=\frac{1}{8}\left\{\eta^{2}\left(1+\cos \theta_{u}\right)^{2}+4(1-\eta)^{2} \sin ^{4}\left(\theta_{v} / 2\right)\right.} \\
+4 \sin ^{4}\left(\theta_{u} / 2\right)+8(1-\eta) \eta \cos ^{2}\left(\theta_{u} / 2\right) \sin ^{2}\left(\theta_{v} / 2\right) \\
\left.+4 \eta\left(1+\cos \theta_{u}\right) \cos \left(2 \psi_{u}\right) \sin ^{2}\left(\theta_{u} / 2\right)\right\}
\end{gathered}
$$

The subsystem purity for the state $\rho^{\prime \prime}$ reads

$$
\begin{aligned}
\mathcal{P}\left(\rho^{\prime \prime}\right)= & \frac{1}{4}\left\{3-\eta(2-\eta)+\frac{1}{2}(1-\eta)^{2}\right. \\
& \left.\times\left[\left(1-\cos \phi_{-}\right) \cos \theta_{+}+\left(1+\cos \phi_{-}\right) \cos \theta_{-}\right]\right\},
\end{aligned}
$$

where

$$
\phi_{ \pm}:=\phi_{u} \pm \phi_{v}, \quad \theta_{ \pm}:=\theta_{u} \pm \theta_{v} .
$$

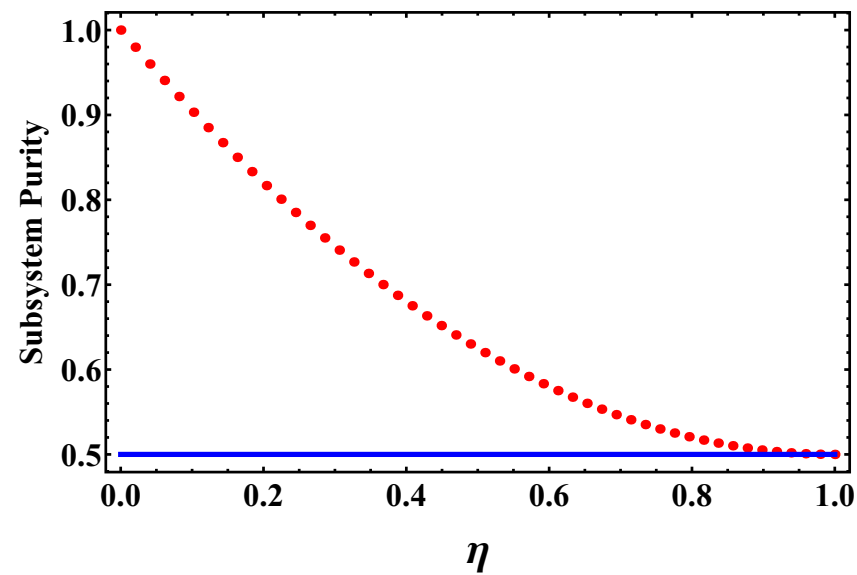

FIG. 2. Subsystem purity versus $\eta$ without feedback action (dotted red line) and in the presence of feedback action with $\theta_{+}=-\theta_{-}=\pi$ (solid blue line).

Taking the partial derivatives of (24) with respect to $\theta_{+}, \theta_{-}$, and $\phi_{-}$and setting them equal to zero, we arrive at the following equations:

$$
\begin{aligned}
\left(\cos \phi_{-}-1\right) \sin \theta_{+} & =0, \\
\left(\cos \theta_{+}-\cos \theta_{-}\right) \sin \phi_{-} & =0, \\
\left(\cos \phi_{-}+1\right) \sin \theta_{-} & =0,
\end{aligned}
$$

which have a set of solutions

$$
\begin{aligned}
& \left\{\theta_{+}=0, \phi_{-}=-\pi\right\}, \quad\left\{\theta_{+}=0, \phi_{-}=\pi\right\}, \\
& \left\{\theta_{+}=0, \theta_{-}=0\right\}, \quad\left\{\theta_{-}=0, \phi_{-}=0\right\},
\end{aligned}
$$

which leads to the same amount of $\mathcal{P}$ without feedback. The other set of solutions of (26),

$$
\begin{aligned}
& \left\{\theta_{+}=-\pi, \theta_{-}=-\pi\right\}, \quad\left\{\theta_{+}=-\pi, \theta_{-}=\pi\right\}, \\
& \left\{\theta_{+}=\pi, \theta_{-}=-\pi\right\}, \quad\left\{\theta_{+}=\pi, \theta_{-}=\pi\right\},
\end{aligned}
$$

leads to constant subsystem purity equal to $1 / 2$ (minimum obtainable value) for any value of $\eta$ (and arbitrary value of $\left.\phi_{-}\right)$. All the values in (28) give the density operator

$$
\rho^{\prime \prime}=\frac{1}{2}\left(\begin{array}{cccc}
1 & 0 & 0 & \eta e^{-2 i\left(\phi_{u}+\psi_{u}\right)} \\
0 & 0 & 0 & 0 \\
0 & 0 & 0 & 0 \\
\eta e^{2 i\left(\phi_{u}+\psi_{u}\right)} & 0 & 0 & 1
\end{array}\right),
$$

whose concurrence results

$$
C\left(\rho^{\prime \prime}\right)=\eta \text {. }
$$

The results for the subsystem purity and concurrence are displayed in Figs. 2 and 3, respectively, which show that the behavior of purity and concurrence versus $\eta$ is consistent. This is also confirmed by numerical analysis of concurrence $C\left(\eta, \phi_{-}, \theta_{u}, \theta_{v}\right)$ (whose results do not depend on $\phi_{+}, \psi_{u}$, and $\psi_{v}$ ) and maximized by taking 11 values for $\eta$ (varying it from 0 to 1 in steps of 0.1 ) and 61 values for $\phi_{-}, \theta_{u}$, and $\theta_{v}$ (varying them from 0 to $2 \pi$ in steps of $\pi / 30$ ). 


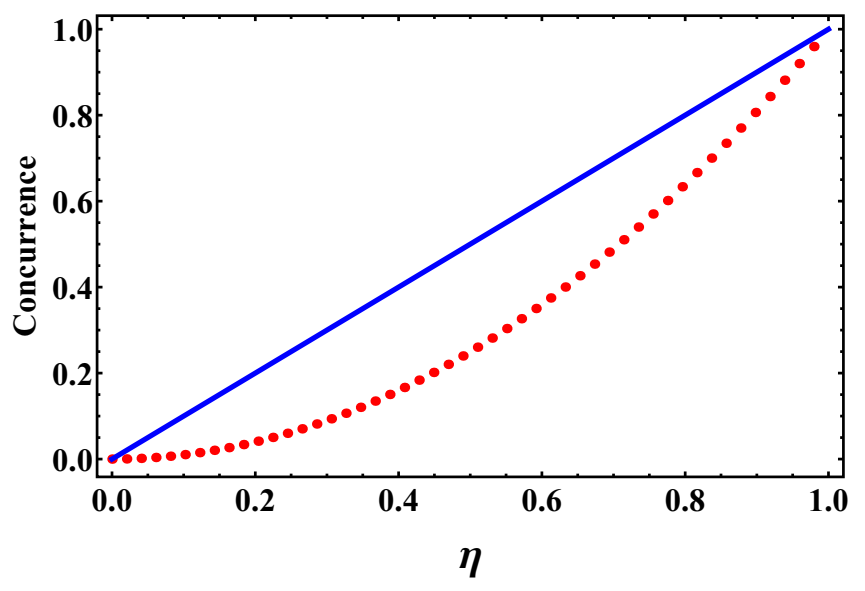

FIG. 3. Concurrence versus $\eta$ without feedback action (dotted red line) and in the presence of feedback action with $\theta_{+}=-\theta_{-}=\pi$ (solid blue line).

\section{OPTIMALITY OF FEEDBACK ACTION}

It is known that the same quantum channel can have many (actually infinite many) Kraus decompositions and each one can be interpreted as a given measurement performed on the environment to gain information about the system [6]. Hence, in this section we will check the optimality of feedback action on the unitarily equivalent Kraus representation of the map (1). To this end, first notice that the Kraus representation provided in (2) is canonical, i.e., $\operatorname{Tr}\left(K_{i}^{\dagger} K_{j}\right) \propto \delta_{i j}$. Then, restricting the discussion to canonical Kraus operators, we should consider new Kraus operators

$$
\tilde{K}_{i}=\sum_{j=1}^{4} V_{i j} K_{j}
$$

obtainable by linear combination of the old ones through a unitary matrix $V=V_{A} \otimes V_{B}$, in which $V_{A}, V_{B} \in \mathrm{SU}(2)$ and similarly to (13) can be parametrized as

$$
\begin{aligned}
V_{A} & =\left(\begin{array}{cc}
\alpha & \beta \\
-\beta^{*} & \alpha^{*}
\end{array}\right), \\
V_{B} & =\left(\begin{array}{cc}
\alpha^{\prime} & \beta^{\prime} \\
-\beta^{* *} & \alpha^{\prime *}
\end{array}\right),
\end{aligned}
$$

with $|\alpha|^{2}+|\beta|^{2}=\left|\alpha^{\prime}\right|^{2}+\left|\beta^{\prime}\right|^{2}=1$. Explicitly we have

$$
\begin{aligned}
& \tilde{K}_{1}=\alpha \alpha^{\prime} K_{1}+\alpha \beta^{\prime} K_{2}+\alpha^{\prime} \beta K_{3}+\beta \beta^{\prime} K_{4}, \\
& \tilde{K}_{2}=-\alpha \beta^{\prime *} K_{1}+\alpha \alpha^{\prime *} K_{2}-\beta \beta^{*} K_{3}+\beta \alpha^{\prime *} K_{4}, \\
& \tilde{K}_{3}=-\alpha^{\prime} \beta^{*} K_{1}-\beta^{\prime} \beta^{*} K_{2}+\alpha^{\prime} \alpha^{*} K_{3}+\beta^{\prime} \alpha^{*} K_{4}, \\
& \tilde{K}_{4}=\beta^{*} \beta^{*} K_{1}-\beta^{*} \alpha^{\prime *} K_{2}-\alpha^{*} \beta^{* *} K_{3}+\alpha^{*} \alpha^{\prime *} K_{4} .
\end{aligned}
$$

This means that we can now describe the dynamics of the density matrix in the presence of feedback as

$$
\rho \mapsto \tilde{\rho}^{\prime \prime}=\sum_{j=1}^{4}\left(U_{j} \tilde{K}_{j}\right) \rho\left(U_{j} \tilde{K}_{j}\right)^{\dagger} .
$$

The expression of $\tilde{\rho}^{\prime \prime}$ is too cumbersome to be reported here. However, by computing its subsystem purity, the surprising aspect is that it becomes a function of only $\{\alpha, \beta\}$. Actually it reads

$$
\mathcal{P}\left(\tilde{\rho}^{\prime \prime}\right)=\frac{1}{8}\left(4+P_{1}^{2}+\left|P_{2}\right|^{2}\right),
$$

in which

$$
\begin{aligned}
& P_{1}:=(1-\eta)\left(\cos \theta_{u}+\cos \theta_{v}\right) \\
&+ 2 \sqrt{(1-\eta) \eta} \sin \theta_{u}\left[\sin \psi_{u} \Im\left(\alpha \beta^{*}\right)-\cos \psi_{u} \Re\left(\alpha \beta^{*}\right)\right] \\
&-2 \sqrt{(1-\eta) \eta} \sin \theta_{v}\left[\sin \psi_{v} \Im\left(\alpha \beta^{*}\right)-\cos \psi_{v} \Re\left(\alpha \beta^{*}\right)\right], \\
& P_{2}:=(1-\eta) \sin \theta_{v}+(1-\eta) e^{-i\left(\phi_{u}-\phi_{v}\right)} \sin \theta_{u} \\
&-\alpha \beta^{*} \sqrt{(1-\eta) \eta}\left(1-\cos \theta_{u}\right) e^{-i\left(\phi_{u}-\phi_{v}-\psi_{u}\right)} \\
&+\alpha^{*} \beta \sqrt{(1-\eta) \eta}\left(1+\cos \theta_{u}\right) e^{-i\left(\phi_{u}-\phi_{v}+\psi_{u}\right)} \\
&+\alpha \beta^{*} \sqrt{(1-\eta) \eta}\left(1-\cos \theta_{v}\right) e^{i \psi_{v}} \\
&-\alpha^{*} \beta \sqrt{(1-\eta) \eta}\left(1+\cos \theta_{v}\right) e^{-i \psi_{v}} .
\end{aligned}
$$

It is obvious that the minimum $1 / 2$ of (35) is achieved when

$$
P_{1}=0, \quad P_{2}=0 \text {. }
$$

The quantity (36) vanishes when $\theta_{u}=0$ and $\theta_{v}=\pi$, which leads to $\theta_{-}=\pi$ and $\theta_{+}=-\pi$. With these values, the quantity (37) vanishes with

$$
\phi_{u}+\psi_{u}=\pi+\phi_{v}-\psi_{v}-2 \xi_{\alpha \beta},
$$

where

$$
\xi_{\alpha \beta}:=\xi_{\alpha}-\xi_{\beta}
$$

with

$$
\begin{aligned}
& \alpha=r_{\alpha} e^{i \xi_{\alpha}}, \\
& \beta=r_{\beta} e^{i \xi_{\beta}},
\end{aligned}
$$

satisfying $r_{\beta}=\sqrt{1-r_{\alpha}^{2}}$. Therefore, in this case, having fixed $\theta_{-}=\pi$ and $\theta_{+}=-\pi$, the concurrence $C\left(\tilde{\rho}^{\prime \prime}\right)$ remains a function of four parameters, i.e., $C\left(\eta, r_{\alpha}, \xi_{\alpha \beta}, \phi_{v}-\psi_{v}\right)$.

In order to find the maximum of concurrence over these four parameters and give a comparison with the concurrence of canonical Kraus operators (30), we perform a numerical maximization over $\eta, r_{\alpha}, \xi_{\alpha \beta}$, and $\phi_{v}-\psi_{v}$. This is done by choosing 11 values for $\eta$ and for $r_{\alpha}$ (varying them from 0 to 1 in steps of 0.1 ) and 61 values for $\xi_{\alpha \beta}$ and for $\phi_{v}-\psi_{v}$ (varying them from 0 to $2 \pi$ in steps of $\pi / 30$ ). For any values of $\eta$, we obtain the maximum of concurrence over another $61^{2} \times 11$ points. The numerical results show that the optimal concurrence is exactly the same as the one obtained in the canonical scenario, i.e., for $r_{\alpha}=1$. Examples of numerical results are reported in Fig. 4. Taking into account the results of this and the previous section (i.e., optimal feedback achieved for $\left\{\theta_{-}=\pi, \theta_{+}=-\pi\right\}$ ) we end up with the following optimal 


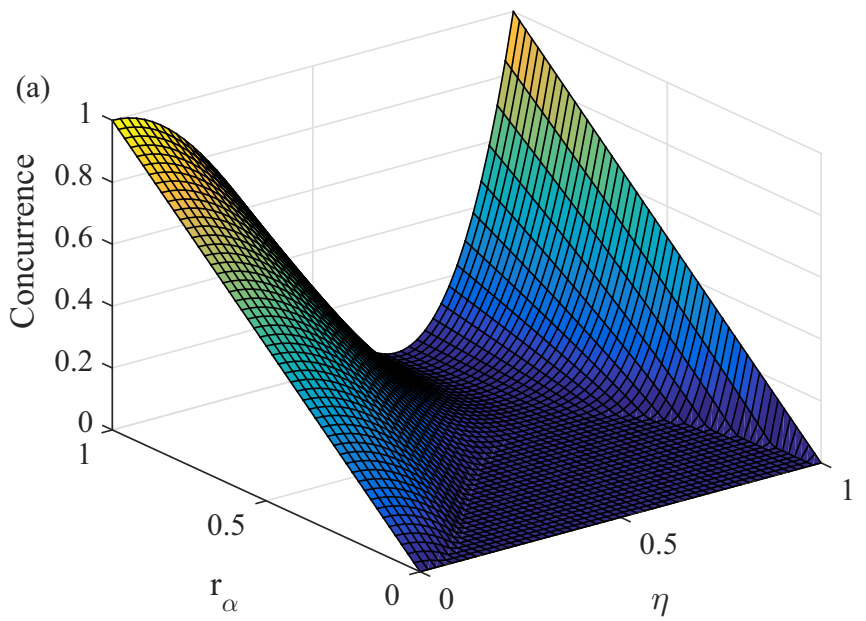

where $q$ is a generic complex number such that $|q| \leqslant 1$. In the computational basis $\mathfrak{B}$ and in the absence of feedback action, the state $\rho_{q}^{\prime}$ resulting from Eq. (1) reads

$$
\rho_{q}^{\prime}=\frac{1}{2}\left(\begin{array}{cccc}
\eta^{2} & 0 & 0 & q \eta \\
0 & \eta(1-\eta) & 0 & 0 \\
0 & 0 & \eta(1-\eta) & 0 \\
q^{*} \eta & 0 & 0 & 2+\eta(\eta-2)
\end{array}\right) .
$$

Its subsystem purity is the same as Eq. (8) but its concurrence now depends on $|q|$. On the other hand, the matrix elements of $\rho_{q}^{\prime \prime}$ in the basis $\mathfrak{B}$ after applying (11), with (12) and (13), on the initial state (43) result:

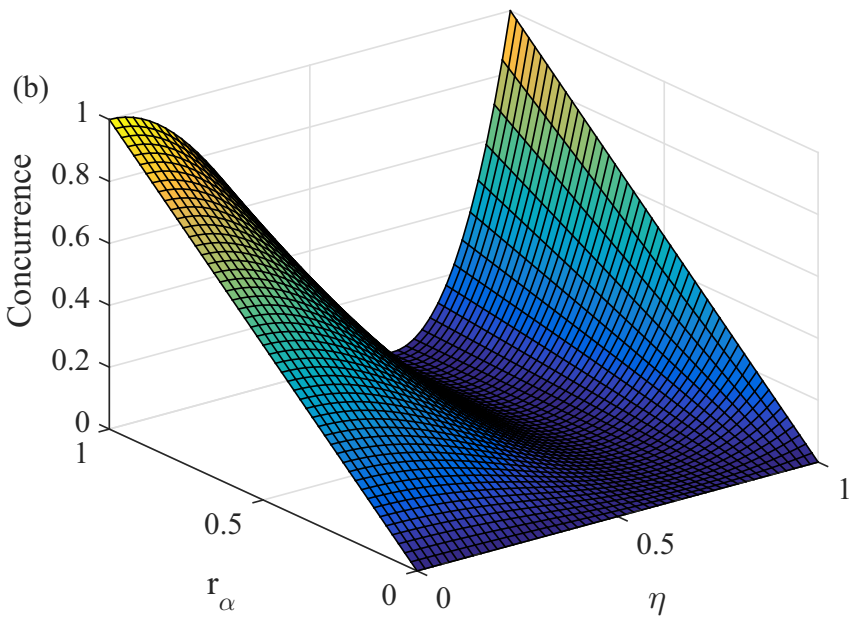

FIG. 4. Numerical results for concurrence under conditions (38), i.e., $\theta_{u}=0$ and $\theta_{v}=\pi$ (or $\theta_{-}=\pi$ and $\theta_{+}=-\pi$ ), for (a) $\xi_{\alpha \beta}=\frac{\pi}{2}$ and $\phi_{v}-\psi_{v}=\frac{\pi}{3}$ and (b) $\xi_{\alpha \beta}=0$ and $\phi_{v}-\psi_{v}=\pi$.

local unitaries characterizing the feedback action in (12):

$$
\begin{aligned}
& u=\left(\begin{array}{cc}
e^{-i\left(\phi_{u}+\psi_{u}\right) / 2} & 0 \\
0 & e^{i\left(\phi_{u}+\psi_{u}\right) / 2}
\end{array}\right), \\
& v=\left(\begin{array}{cc}
0 & -e^{-i\left(\phi_{v}-\psi_{v}\right) / 2} \\
e^{i\left(\phi_{v}-\psi_{v}\right) / 2} & 0
\end{array}\right),
\end{aligned}
$$

with arbitrary $\phi_{u}+\psi_{u}$ and $\phi_{v}-\psi_{v}$.

\section{REPEATED FEEDBACK ACTION}

Returning to Eq. (29), we may observe that the matrix representation of $\rho^{\prime \prime}$ has nonzero entries where also $\rho$ of Eq. (5) has. Hence we may argue that the devised feedback action is optimal also starting from (29).

Then we repeat the analysis of Secs. III and IV starting from a state

$$
\rho_{q}=\frac{1}{2}\left(\begin{array}{cccc}
1 & 0 & 0 & q \\
0 & 0 & 0 & 0 \\
0 & 0 & 0 & 0 \\
q^{*} & 0 & 0 & 1
\end{array}\right)
$$

$$
\begin{aligned}
{\left[\rho_{q}^{\prime \prime}\right]_{11}=} & \frac{1}{2}\left\{\left(1-\eta^{2}\right) \cos ^{2}\left(\theta_{v} / 2\right)+\eta^{2} \sin ^{4}\left(\theta_{u} / 2\right)\right. \\
& +2 \eta(1-\eta) \cos ^{2}\left(\theta_{v} / 2\right) \sin ^{2}\left(\theta_{u} / 2\right)+\cos ^{2}\left(\theta_{u} / 2\right) \\
& \left.+\eta\left(1+\cos \theta_{u}\right) \Re\left(q e^{-2 i \psi_{u}}\right) \sin ^{2}\left(\theta_{u} / 2\right)\right\}, \\
{\left[\rho_{q}^{\prime \prime}\right]_{12}=} & \frac{1}{8}\left\{e^{-i \phi_{v}}(1-\eta)^{2} \sin \theta_{v}\left[\cos \theta_{v}+\frac{1-\eta \cos \theta_{u}}{1-\eta}\right]\right. \\
& +e^{-i \phi_{u}} \sin \theta_{u}\left[(1-\eta)\left(1-\eta \cos \theta_{v}\right)+\left(1+\eta^{2}\right) \cos \theta_{u}\right. \\
& \left.-2 \eta \cos \theta_{u} \Re\left(q e^{-2 i \psi_{u}}\right)-2 i \eta \Im\left(q e^{\left.-2 i \psi_{u}\right)}\right\}\right\}, \quad[46) \\
{\left[\rho_{q}^{\prime \prime}\right]_{14}=} & \frac{1}{8} e^{-2 i\left(\phi_{u}+\psi_{u}\right)}\left\{\eta q\left(1+\cos \theta_{u}\right)^{2}\right. \\
+ & 4 e^{2 i \psi_{u}}\left[q^{*} \eta e^{2 i \psi_{u}}+\left(1+\rho^{\prime \prime}\right]_{12},\right. \\
+ & 2 e^{2 i\left(\phi_{u}-\phi_{v}+\psi_{u}\right)}(1-\eta)^{2}\left(1+\cos ^{2}\left(\theta_{u} / 2\right)\right] \sin ^{2}\left(\theta_{u} / 2\right) \sin ^{2}\left(\theta_{v} / 2\right) \\
& \left.-2 e^{i\left(\phi_{u}-\phi_{v}+2 \psi_{u}\right)} \eta(1-\eta) \sin _{u} \sin _{v}\right\}, \\
& \frac{1}{8}\left\{4(1-\eta) \eta \cos ^{2}\left(\theta_{u} / 2\right) \cos ^{2}\left(\theta_{v} / 2\right)\right. \\
& \left.+\left[1+\rho_{q}^{\prime \prime}\right]_{22}-2 \eta \Re\left(q e^{-2 i \psi_{u}}\right)\right] \sin ^{2} \theta_{u} \\
& \left.+2(1-\eta)\left[1-\eta \cos \theta_{u}+(1-\eta) \cos \theta_{v}\right] \sin ^{2}\left(\theta_{v} / 2\right)\right\},
\end{aligned}
$$

$$
\begin{aligned}
{\left[\rho_{q}^{\prime \prime}\right]_{23}=} & \frac{1}{8}\left\{\left[1+\eta^{2}-2 \eta \Re\left(q e^{-2 i \psi_{u}}\right)\right] \sin ^{2} \theta_{u}\right. \\
& -(1-\eta) \sin \theta_{v}\left[2 \eta \cos \left(\phi_{u}-\phi_{v}\right) \sin \theta_{u}\right. \\
& \left.\left.-(1-\eta) \sin \theta_{v}\right]\right\}
\end{aligned}
$$

$$
\begin{aligned}
{\left[\rho_{q}^{\prime \prime}\right]_{24}=} & \frac{1}{8}\left(e ^ { - i \phi _ { u } } \operatorname { s i n } \theta _ { u } \left\{(1-\eta)\left(1+\eta \cos \theta_{v}\right)\right.\right. \\
& \left.-\left[1+\eta^{2}-2 \eta \Re\left(q e^{-2 i \psi_{u}}\right)\right] \cos \theta_{u}+2 i \eta \Im\left(q e^{-2 i \psi_{u}}\right)\right\} \\
& \left.+e^{-i \phi_{v}}(1-\eta)\left[1+\eta \cos \theta_{u}-(1-\eta) \cos \theta_{v}\right] \sin \theta_{v}\right)
\end{aligned}
$$

$$
\begin{aligned}
& {\left[\rho_{q}^{\prime \prime}\right]_{33}=\left[\rho_{q}^{\prime \prime}\right]_{22},} \\
& {\left[\rho_{q}^{\prime \prime}\right]_{34}=\left[\rho_{q}^{\prime \prime}\right]_{24},}
\end{aligned}
$$




$$
\begin{aligned}
{\left[\rho_{q}^{\prime \prime}\right]_{44}=} & \frac{1}{8}\left\{\eta^{2}\left(1+\cos \theta_{u}\right)^{2}+4(1-\eta)^{2} \sin ^{4}\left(\theta_{v} / 2\right)\right. \\
& +4 \sin ^{4}\left(\theta_{u} / 2\right)+8(1-\eta) \eta \cos ^{2}\left(\theta_{u} / 2\right) \sin ^{2}\left(\theta_{v} / 2\right) \\
& \left.+4 \eta\left(1-\cos ^{2} \theta_{u}\right) \Re\left(q e^{-2 i \psi_{u}}\right)\right\} .
\end{aligned}
$$

For the state $\rho_{q}^{\prime \prime}$, the subsystem purity turns out to be the same as (24), i.e., not dependent on $q$. This leads us to conclude that also for $|q|<1$ the optimal feedback is achieved by $\left\{\theta_{-}=\right.$ $\left.\pi, \theta_{+}=-\pi\right\}$ and hence (42). With this, the state after feedback action reads, in the basis $\mathfrak{B}$,

$$
\rho_{q}^{\prime \prime}=\frac{1}{2}\left(\begin{array}{cccc}
1 & 0 & 0 & q \eta e^{-2 i\left(\phi_{u}+\psi_{u}\right)} \\
0 & 0 & 0 & 0 \\
0 & 0 & 0 & 0 \\
q^{*} \eta e^{2 i\left(\phi_{u}+\psi_{u}\right)} & 0 & 0 & 1
\end{array}\right) .
$$

Its concurrence results:

$$
C\left(\rho_{q}^{\prime \prime}\right)=|q| \eta
$$

The optimality of this result is confirmed by numerical investigations over noncanonical Kraus decompositions (31). Similarly to Sec. IV, we have maximized the concurrence $C\left(\rho_{q}^{\prime \prime}\right)$ over the parameters $r_{\alpha}, \xi_{\alpha \beta}$, and $\phi_{v}-\psi_{v}$, this time for each pair of values of $\eta$ and $q$. This has been done by choosing 11 values for $\eta$, for $|q|$, and for $r_{\alpha}$ (varying them between 0 and 1 in steps of 0.1 ) and 61 values for $\xi_{\alpha \beta}$ and for $\phi_{v}-\psi_{v}$ (varying them from 0 to $2 \pi$ in steps of $\pi / 30$ ). For any pair of $\eta$ and $|q|$ the maximum concurrence has been obtained over another $61^{2} \times 11$ points. The numerical results show that the optimal concurrence is exactly (56), i.e., the one obtained in the canonical scenario $\left(r_{\alpha}=1\right)$.

Due to the above results, we can consider repeated applications of the map without feedback, giving

$$
\rho^{\prime(n)}=\frac{\eta^{n}}{2}\left(\begin{array}{cccc}
\eta^{n} & 0 & 0 & 1 \\
0 & 1-\eta^{n} & 0 & 0 \\
0 & 0 & 1-\eta^{n} & 0 \\
1 & 0 & 0 & \frac{2}{\eta^{n}}-\left(2-\eta^{n}\right)
\end{array}\right) \text {, }
$$

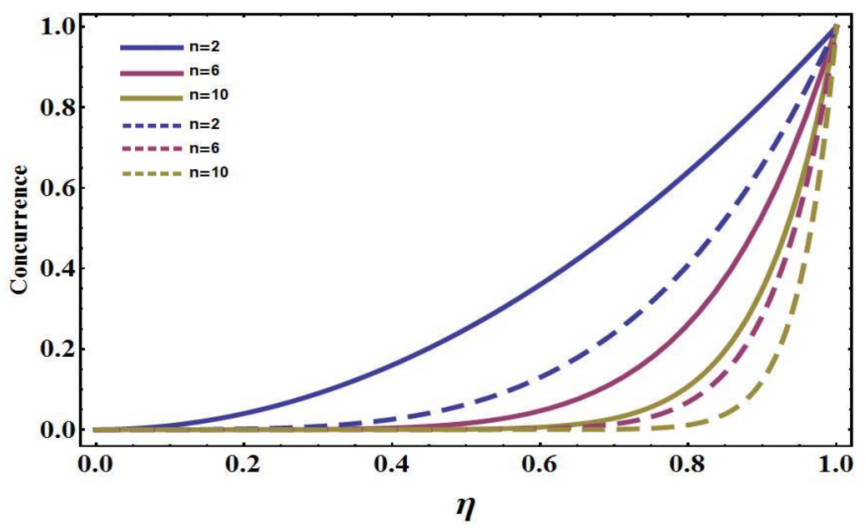

FIG. 5. Concurrence versus $\eta$ for different number $n$ of applications of the amplitude damping map, without feedback action (dashed line) and with feedback action (solid line).

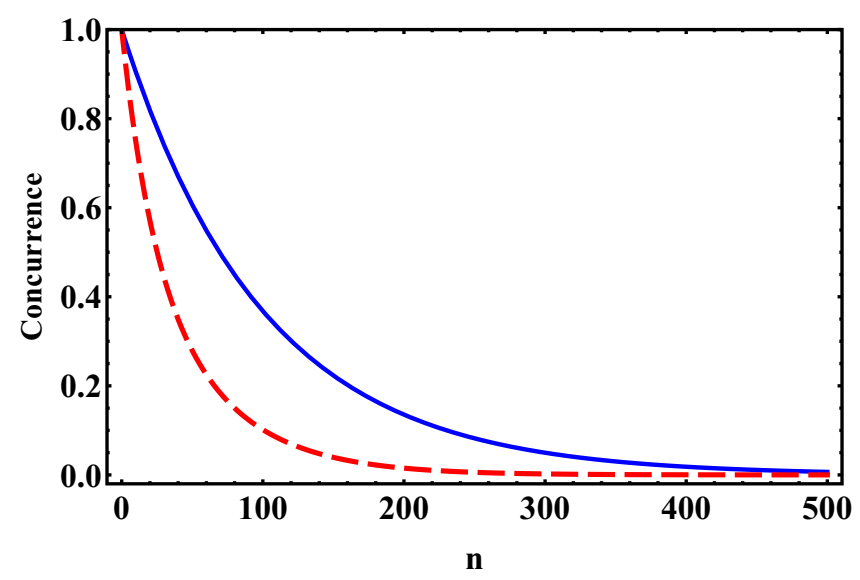

FIG. 6. Concurrence vs $n$ without feedback action (dashed red line) and with feedback action (solid blue line) for $\eta=0.99$.

where $n$ is the number of map applications, as well as repeated applications of the map with feedback giving

$$
\rho^{\prime \prime(n)}=\frac{1}{2}\left(\begin{array}{cccc}
1 & 0 & 0 & \eta^{n} e^{-2 n i\left(\phi_{u}+\psi_{u}\right)} \\
0 & 0 & 0 & 0 \\
0 & 0 & 0 & 0 \\
\eta^{n} e^{2 n i\left(\phi_{u}+\psi_{u}\right)} & 0 & 0 & 1
\end{array}\right) .
$$

The corresponding concurrences

$$
\begin{aligned}
C\left(\rho^{\prime(n)}\right)= & \frac{\eta^{n}}{2}\left[\sqrt{\left(\eta^{n}-2\right) \eta^{n}+3+2 \sqrt{\left(\eta^{n}-2\right) \eta^{n}+2}}\right. \\
& -\sqrt{\left(\eta^{n}-2\right) \eta^{n}+3-2 \sqrt{\left(\eta^{n}-2\right) \eta^{n}+2}} \\
& \left.-2\left(1-\eta^{n}\right)\right]
\end{aligned}
$$

and

$$
C\left(\rho^{\prime \prime(n)}\right)=\eta^{n}
$$

are reported in Fig. 5. There we can see that the advantage of feedback tends to persist only at sufficiently high values of $\eta$, by increasing $n$.

Finally, to have an idea of what would happen in the continuous-time limit, we could consider $t=n \Delta t$ with $\Delta t$ quite small and $n$ positive integers. Since in the continuoustime description of the amplitude damping process the damping rate always appears multiplied by the time (see, e.g., [12]), we can argue that the role of $\Delta t$ in our context is played by $1-\eta$. The closer $\eta$ is to 1 , the smaller $\Delta t$ is. Thus having fixed the value of $\eta$, we have plotted the concurrence vs $n$ in Fig. 6, which shows an exponential (smooth half-life-type) decay. It also shows that feedback can slow down such a decay, but not prevent it from occurring.

\section{CONCLUSION}

We have addressed the problem of correcting errors intervening in two qubits dissipating into their own environments by resorting to local feedback actions with the aim of preserving 
as much as possible the initial amount of entanglement. Optimal control is found by first gaining insights from the subsystem purity and then by numerical analysis of the concurrence. This is tantamount to a double optimization on the actuation and on the measurement processes. The results are obtained for a single shot. The results, although obtained with the help of numerics, are analytically clear and can be summarized by Eqs. (11) and (12) with (42).

Our results could be helpful in designing experiments of entanglement control, particularly in settings such as cavity QED or solid-state base qubits. As an example we could conceive of each qubit as a two-level atom placed at the focus of a parabolic mirror so that the spontaneously emitted light emerges as a beam and let the beam impinge upon a beam splitter with the transmitted beam subjected to homodyne detection [13]. Then our $\eta$ becomes the product of the spontaneous emission rate with the beam-splitter transmissivity. The feedback can be implemented through local electromagnetic fields acting on the atoms. Another example could be provided by two superconducting qubits, each one placed in its own three-dimensional cavity and dispersively coupled to it (see, e.g., [14]). States of the qubit shift the energy of the superconducting cavity and vice versa via their coupling. The product of qubit decay rate with cavity damping rate plays the role of our $\eta$. Furthermore, the outputs of cavities are monitored and the cavity driving fields implement the feedback. ${ }^{1}$ Yet other realizations could be inspired by the review article in [16].

Finally, it is worth remarking that the feedback strategy employed here is in the same spirit as direct feedback [17] in that it does not involve processing the information obtained from the system in order to estimate its state. On the other hand, in the context of repeated map applications and particularly in the continuous-time analysis of the problem, optimization of feedback action should also involve Bayesian (state-estimation-based) strategies and an extension to two qubits of the analysis for single-qubit control performed in Ref. [18] would be welcome.

\section{ACKNOWLEDGMENT}

A.N. would like to thank the University of Camerino for kind hospitality during the early stage of this work.

\footnotetext{
${ }^{1}$ A similar system was employed to show entanglement stabilization by feedback [15].
}

[1] R. Horodecki, P. Horodecki, M. Horodecki, and K. Horodecki, Rev. Mod. Phys. 81, 865 (2009).

[2] J. Opt. B 7(10) (2005), special issue on quantum control, edited by S. Mancini, V. I. Man'ko, and H. M. Wiseman.

[3] H. Rabitz, New J. Phys. 11, 105030 (2009).

[4] D. Dong and I. R. Petersen, IET Control Theory Appl. 4, 2651 (2010).

[5] B. Qi and L. Guo, Syst. Control Lett. 59, 333 (2010).

[6] K. Kraus, States, Effects and Operations: Fundamental Notions of Quantum Theory (Wiley, New York, 1991).

[7] K. Jacobs, Proc. 6th Asian Control Conf., p. 35 (2006), arXiv:quant-ph/0605015.

[8] S. Mancini and H. M. Wiseman, Phys. Rev. A 75, 012330 (2007); A. Serafini and S. Mancini, Phys. Rev. Lett. 104, 220501 (2010).

[9] S. Mancini and J. Wang, Eur. Phys. J. D 32, 257 (2005).

[10] M. Gregoratti and R. F. Werner, J. Mod. Opt. 50, 915 (2003); L. Memarzadeh, C. Cafaro, and S. Mancini, J. Phys. A: Math. Theor. 44, 045304 (2011); L. Memarzadeh, C. Macchiavello, and S. Mancini, New J. Phys. 13, 103031 (2011).
[11] W. K. Wootters, Phys. Rev. Lett. 80, 2245 (1998).

[12] H. P. Breuer and F. Petruccione, The Theory of Open Quantum Systems (Oxford University Press, Oxford, 2002).

[13] J. Gambetta and H. M. Wiseman, Phys. Rev. A 64, 042105 (2001).

[14] A. Wallraff, D. I. Schuster, A. Blais, L. Frunzio, R.-S. Huang, J. Majer, S. Kumar, S. M. Girvin, and R. J. Schoelkopf, Nature (London) 431, 162 (2004).

[15] S. Shankar, M. Hatridge, Z. Leghtas, K. M. Sliwa, A. Narla, U. Vool, S. M. Girvin, L. Frunzio, M. Mirrahimi, and M. H. Devoret, Nature (London) 504, 419 (2013).

[16] J. Zhang, Y.-x. Liu, R.-B. Wu, K. Jacobs, and F. Nori, arXiv: 1407.8536 .

[17] H. M. Wiseman and G. J. Milburn, Phys. Rev. Lett. 70, 548 (1993); H. M. Wiseman, Phys. Rev. A 49, 2133 (1994).

[18] H. M. Wiseman, S. Mancini, and J. Wang, Phys. Rev. A 66, 013807 (2002). 